\title{
Turbulence and particle acceleration in collisionless supernovae remnant shocks
}

\section{Cosmic-ray transport}

\author{
A. Marcowith ${ }^{1}$, M. Lemoine ${ }^{2}$, and G. Pelletier ${ }^{3}$ \\ ${ }^{1}$ Centre d'Études Spatiales et du Rayonnement, CNRS, Université Paul Sabatier Toulouse 3, 9 avenue du Colonel Roche, \\ 31028 Toulouse Cedex, France \\ e-mail: Alexandre.Marcowith@cesr.fr \\ 2 Institut d'Astrophysique de Paris, UMR 7095 CNRS, Université Pierre \& Marie Curie, 98bis boulevard Arago, 75014 Paris, France \\ e-mail: lemoine@iap.fr \\ ${ }^{3}$ Laboratoire d'Astrophysique de Grenoble, CNRS, Université Joseph Fourier II, BP 53, 38041 Grenoble, \\ and Institut Universitaire de France, France \\ e-mail: Guy.Pelletier@obs.ujf-grenoble.fr
}

Received 20 December 2005 / Accepted 16 March 2006

\section{ABSTRACT}

\begin{abstract}
Supernovae remnant shock waves could be at the origin of cosmic rays up to energies in excess of the knee $\left(E \simeq 3 \times 10^{15} \mathrm{eV}\right)$ if the magnetic field is efficiently amplified by the streaming of accelerated particles in the shock precursor. This paper follows up on a previous paper (Pelletier et al. 2006, A\&A, in press) which derived the properties of the MHD turbulence so generated, in particular its anisotropic character, its amplitude and its spectrum. In the present paper, we calculate the diffusion coefficients, also accounting for compression through the shock, and show that the predicted three-dimensional turbulence spectrum $k_{\perp} S_{3 \mathrm{~d}}\left(k_{\|}, k_{\perp}\right) \propto k_{\|}^{-1} k_{\perp}^{-\alpha}$ (with $k_{\|}$and $k_{\perp}$ the wavenumber components along and perpendicular to the shock normal) generally leads to Bohm diffusion in the parallel direction. However, if the anisotropy is constrained by a relation of the form $k_{\|} \propto k_{\perp}^{2 / 3}$, which arises when the turbulent energy cascade occurs at a constant rate independent of scale, then the diffusion coefficient loses its Bohm scaling and scales as in isotropic Kolmogorov turbulence. We show that these diffusion coefficients allow to account for X-ray observations of supernova remnants. This paper also calculates the modification of the Fermi cycle due to the energy lost by cosmic rays in generating upstream turbulence and the concomittant steepening of the energy spectrum. Finally we confirm that cosmic rays can produced an amplified turbulence in young $\mathrm{SNr}$ during their free expansion phase such that the maximal energy is close to the knee and the spectral index is close to 2.3 in the warm phase of the interstellar medium.
\end{abstract}

Key words. acceleration of particles - shock waves - turbulence - ISM: supernova remnants

\section{Introduction}

Supernovae (SN) blastwaves are probable sites of cosmic ray (CR) acceleration up to energies of a few $10^{14} \mathrm{eV}$ as discussed by Lagage \& Césarsky (1983). The cosmic rays are accelerated through the diffusive shock acceleration mechanism which invokes upstream and downstream turbulence and whose efficiency is fully determined by the transport properties of the cosmic rays in these regions (see reviews by Drury 1983; Jones \& Ellison 1991; and more recently by Drury 2001; Hillas 2005). The maximum cosmic-ray energy attainable in supernova remnants (SNR) scales as the product of magnetic field and size of the accelerator; the above Lagage \& Césarsky estimate for the maximum energy assumes that the fluctuating part of the magnetic field $\delta B$ has saturated at a level of the order of the far upstream interstellar value $B_{\infty}$ (McKenzie \& Völk 1982). However, recent observational and theoretical studies have indicated that young SNR are likely sites of efficient magnetic field amplification with a fluctuating component reaching levels $\gg B_{\infty}$.

High resolution X-ray observations of young SNR have revealed the presence of sharp external rims associated with the blastwave, produced by synchrotron radiation of relativistic electrons (Gotthelf et al. 2001; Hwang et al. 2002; Long et al. 2003; Vink 2004; Ballet 2005). As discussed in Sect. 2 below, the smallness of the rim size implies a downstream diffusion coefficient for the relativistic electrons close to its Bohm value (Berezhko et al. 2003; Berezhko \& Völk 2004; Parizot et al. 2006) i.e. $D_{\mathrm{e}} \simeq r_{\mathrm{L}} c / 3$. Bell \& Lucek (2001) and Lucek \& Bell (2000) have investigated in some details the non-linear development of the cosmic ray streaming instability and obtained an amplified magnetic field well in excess of $B_{\infty}$. These authors have suggested that the tangled character of the magnetic field implies a CR mean free path of the order of its Larmor radius (the Bohm diffusion regime). Both Bohm diffusion and a strong magnetic field amplification seem necessary to push the maximum cosmic-ray energies up to the CR knee at a few $\mathrm{PeV}$ or even up to the CR ankle at a few EeV (Ptuskin \& Zirakshvilli 2003, 2005). This amplification mechanism then appears essential to our understanding of galactic cosmic-ray phenomenology and theory.

However, previous theoretical work suffers from various limitations. For instance, the streaming of cosmic-rays amplifies forward waves at the same time as it damps backward waves, and the transfer of energy between these two types of waves has not been modeled by Bell \& Lucek (2001). The numerical simulations using coupled "Particle in Cells" and magnetohydrodynamics codes suffer from limited wave number dynamics and a 
crude modeling of the acceleration mechanism, questioning the value obtained for the non-linear saturation level of the magnetic field. The argument in favor of a Bohm diffusion regime is also phenomenological since no MHD theory has rigorously predicted such regime yet (see Casse et al. 2002, and references therein), although Lucek \& Bell (2000) did observe isotropisation of the CR distribution on a timescale of a gyroperiod in their numerical experiments. Finally, the non-resonant instability mechanism uncovered by Bell (2004) has not been taken into account in previous studies. These points have motivated us to investigate in some details the generation of turbulence in the shock precursor by cosmic-ray streaming through both resonant and non-resonant instabilities (Pelletier et al. 2006), hereafter refered as Paper I. We have notably found that the three-dimensional spectrum of upstream turbulence so excited is anisotropic and scales as $S_{3 \mathrm{~d}}\left(k_{\|}, k_{\perp}\right) \propto k_{\|}^{-1} k_{\perp}^{-\alpha-1}$ (with $k_{\|}$along and $k_{\perp}$ perpendicular to the shock normal and to the mean magnetic field), and that the downstream turbulence is further amplified by the jump through the shock.

In the present work, we propose to use the new results of Paper I to investigate the acceleration of cosmic rays in young SNR. In particular, we explicitly calculate the diffusion coefficients both upstream and downstream using Monte-Carlo simulations and analytical quasi-linear theory. We thus provide a connection between the prediction of turbulence spectra and the observations of electron synchrotron radiation. We also apply the results to the Fermi cycle of cosmic rays. Finally we follow in detail the energy budget, accounting in particular for the energy spent in amplifying the upstream magnetic field.

\section{Observations}

\subsection{X-ray and radio observations of the outer rims}

Quite recently the X-ray instrument Chandra has imaged the continuum emission (4 to $6 \mathrm{keV}$ ) in different young supernova remnants like Cas A (Gotthelf et al. 2001), Tycho (Hwang et al. 2002) and SN1006 (Bamba et al. 2003). The X-ray radiation originates from very thin sheets behind the blast wave. The same conclusion have been drawn by XMM-Newton observations of the Kepler SNr (Cassam-Chenaï et al. 2004). The derived physical width of the emission region is less than $4^{\prime \prime}$ or $2 \times 10^{17} \mathrm{~cm}$ in Cas A, for a shock velocity $V_{\mathrm{sh}} \simeq 5000 \mathrm{~km} \mathrm{~s}^{-1}$, less than $4^{\prime \prime}$ or $1.5 \times 10^{17} \mathrm{~cm}$ in Tycho for a shock velocity $V_{\mathrm{sh}} \simeq 4600 \mathrm{~km} \mathrm{~s}^{-1}$. The results for three young $\mathrm{SNr}$ (Cas A, Tycho and Kepler) and two older ones (SN1006 and G347.3-0.5) have been presented in Ballet (2005). The effective rim size may however be overestimated due to projection effects (Berezhko et al. 2003). Ballet (2005) has also shown that this radiation is inconsistent with thermal models in a uniform medium which predict emission everywhere up to the interface, with only a slight maximum at the blast wave. A second argument against thermal models is the rather featureless spectrum in the $1-10 \mathrm{keV}$ band which appears to be inconsistent with the X-ray brightness (see for instance Cassam-Chenaï et al. 2004, in the case of the Kepler SNr). The only other possible source of X-ray radiation is suprathermal particles. Non-thermal bremsstrahlung (by lowenergy suprathermal electrons) could still be compatible with the observed rims for a low thermal gas temperature of $\sim 1-2 \times 10^{6} \mathrm{~K}$ and a high enough target density (Ballet 2005). Another natural mechanism is the synchrotron radiation by ultra-relativistic electrons with energies of few tens of TeV (Vink \& Laming 2003; Vink 2004). The radio synchrotron emission from $\mathrm{GeV}$ electrons is expected to have larger extent as the loss timescale at these energies is much longer. This seems to be indeed the case for Cas A (Gotthelf et al. 2001) and SN1006 (Long et al. 2003) (for this source the rim profile has been plotted in radio and in two X-ray bands showing a systematic sharpening when shifting to the highest frequencies). The case of Tycho $\mathrm{SNr}$ seems to be more complex, here the two rims (radio and X-rays) do not track each other in brightness well (Hwang et al. 2002). We shall discuss in details the physical consequences of these observations on electrons and cosmic rays in Sect. 2.2.

\subsubsection{Constraints from the shock precursor}

The shock precursor region is of prime importance as it is the place where most of the turbulence is expected to be generated. Achterberg et al. (1994) noticed that an upper limit on the precursor size implies an upper limit on the electron mean free path and a lower limit on the level of turbulence. Depending on the scale over which the emissivity drops upstream $\ell_{1 / 2}$, the electron mean free path is $\lambda_{\mathrm{e}} \leq 3\left(V_{\mathrm{sh}} / c\right) \ell_{1 / 2} /\left[a \cos ^{2}\left(\theta_{B n}\right)\right]$ where $\theta_{B n}$ is the obliquity angle between the shock normal and the upstream magnetic field, and $a$ accounts for an emissivity drop; it equals $1 / \log (2)$ in an exponential profile or 3 for a smoother profile where the turbulence is self-generated.

With the help of high resolution radio observations, Achterberg et al. (1994) concluded to a magnetic field amplification of about one order of magnitude compared to the standard interstellar values. This conclusion is however not correct in case of non diffusive transport where supplementary information about the number of upstream shock crossings are required (Ragot 2001). If the magnetic field is purely turbulent upstream, one can replace in the previous expression $\cos ^{2} \theta_{B n}$ by $1 / 3$. This leads to upper limits for $\lambda_{\mathrm{e}}$ of the ordrer of $10^{-3} \mathrm{pc}\left(V_{\mathrm{sh}} / 3000 \mathrm{~km} \mathrm{~s}^{-1}\right)\left(\ell_{1 / 2} / 0.01 \mathrm{pc}\right)$. For instance, Tycho and Kepler have $\ell_{1 / 2} \simeq 7 \times 10^{-3} \mathrm{pc}, \lambda_{\mathrm{e}} \leq 1.1 \times 10^{-3} \mathrm{pc}$, and $V_{\mathrm{sh}} \simeq 5400 \mathrm{~km} \mathrm{~s}^{-1}, \ell_{1 / 2} \simeq 7 \times 10^{-2} \mathrm{pc}, \lambda_{\mathrm{e}} \leq 10^{-2} \mathrm{pc}$ respectively. The diffusion coefficient for radio electrons $D \simeq v \lambda_{\mathrm{e}} / 3$ is limited to $3 \times 10^{25-26} \mathrm{~cm}^{2} \mathrm{~s}^{-1}$. The Bohm value $D_{\text {Bohm }} \simeq$ $6 \times 10^{22}(\mathrm{E} / 1 \mathrm{GeV})(\mathrm{B} / 1 \mu \mathrm{G})^{-1} \mathrm{~cm}^{2} \mathrm{~s}^{-1}$ even if compatible with these constraints only provides loose constraints. In X-rays the emitted radiation must be asymmetrical due to the magnetic field jump at the shock front. The observed X-ray profile is strongly dependent on the projection effects and it appears that most (if not all) of the X-ray photons should come from downstream (Berezhko et al. 2003).

To conclude, it is important to note that the synchrotron interpretation of the radio and $\mathrm{X}$-ray emission is a natural option to explain the high resolution images from X-rays and radio telescopes and the non-thermal spectra by Chandra and XMMNewton. But this model still have some difficulties and should be considered with caution. However, we adopt in the following the synchrotron mechanism as the dominant radiative process in the sharp outer rims.

\subsection{Consequences on the particle acceleration process}

\subsubsection{Electrons}

The size of the X-ray rims downstream the blast wave have important constraints on the relativistic particle transport. For electrons, one can expect that in the loss limited case the size of the rim is set fixed either by advection or by diffusion. In fact, the electron energy is expected to be close to the maximum energy given by $t_{\text {cool }}\left(E_{\max }\right)=t_{\text {acc }}\left(E_{\max }\right) \simeq \kappa / v_{\text {sh }}^{2}$; in that case the advection scale $\ell_{\text {adv }}=t_{\text {cool }} V_{\text {sh }}$ should be close to the diffusive scale 
$\ell_{\text {diff }}=\sqrt{\kappa t_{\text {cool }}}$ (Berezhko et al. 2003; Vink \& Laming 2003). If $E \simeq E_{\max }$, by comparing the advection length with the rim sizes inferred from the observations, one deduces typical magnetic field strengths of the order of a few $100 \mu \mathrm{G}$ in most of all young SNR cited above (Ballet 2005), pointing towards an important magnetic field amplification at the shock precursor by the accelerated particles themselves. Assuming a diffusion coefficient $D=\alpha D_{\text {Bohm }} \simeq \alpha \times 6 \times 10^{24}(\mathrm{E} / 10 \mathrm{TeV})(\mathrm{B} / 100 \mu \mathrm{G})^{-1} \mathrm{~cm}^{2} \mathrm{~s}^{-1}$, with $\alpha \geq 1$, the previous constraints impose $\alpha$ between 1 and 10 (Parizot et al. 2006).

\subsubsection{Cosmic-rays}

In the previous paragraph, we have found that high energy electrons accelerated close to the Bohm value produce synchrotron $\mathrm{X}$-ray profiles compatible with the most recent Chandra observations of young SNr. Likewise, protons and heavier nuclei are prone to diffusive shock acceleration in this highly disordered magnetic field. However, one may expect protons energies to be much higher than few tens of $\mathrm{TeV}$ and have Larmor radii allowing them to explore the largest turbulent scales. At these wavelengths no information is available on the characteristics of the turbulence yet, i.e. the spectral index, the turbulence level. Even if the diffusion coefficient is close to its Bohm value at the energy where the electrons produce synchrotron photons in the 4-6 keV range, there is no observational evidence that it remains true at higher energies. In most of the previous approaches (Bell \& Lucek 2001; Ptuskin \& Zirakshvilli 2003; Berezhko et al. 2003; Bell 2004) only an heuristic argument was used, i.e. the particle mean free path is expected to match the Larmor radius in a completely disordered magnetic field. Numerical experiments have however shown that in the limit of a strongly turbulent magnetic field, in the case of a Kolmogorov turbulence, the diffusion coefficient approaches its Bohm value at large rigidities only, i.e. for Larmor radius close to the maximum turbulence scale (Casse et al. 2002). Parizot et al. (2006) using the X-ray observations from a sample of young SNR have investigated all possibilities testing the spatial transport of cosmic-rays in an isotropic turbulence. They conclude that spectral turbulence indexes smaller than $3 / 2$ (index of the $1 \mathrm{~d}$ spectrum) are to be rejected as they lead to a diffusion coefficient at the maximum proton energies smaller than the Bohm diffusion coefficient. Even if the diffusion regime is close to Bohm, the authors did not find maximum cosmic-ray energies beyond the $\mathrm{CR}$ knee $\left(\simeq 3 \times 10^{15} \mathrm{eV}\right)$, questioning the capability of young $\mathrm{SNr}$ to produce the high energy part of the galactic CR spectrum.

\section{Summary of the results of Paper I}

In order to discriminate between the different transport regimes described above, we have investigated in Paper I in greater detail the turbulence spectrum that results from cosmic-ray interactions in the upstream medium. This work has shown that the turbulence growth occurs as a result of two instabilities. At large distances from the shock front, the non-resonant instability (Bell 2004) that results from the non-zero return current in the thermal plasma, amplifies the turbulence at short wavelengths (as compared to the typical cosmic-ray Larmor radius at that distance from the shock front). At closer distances, cosmicrays of Larmor radius $r_{\mathrm{L}}$ excite resonantly forward turbulent modes of wavenumber $k_{\|}=1 / r_{\mathrm{L}}$ (Bell \& Lucek 2001). These instabilities govern the growth of the parallel forward modes and the concomittant damping of the backward parallel spectrum. While the non-resonant growth saturates when energy is redistributed through non-linear transfers as fast it is input, the resonant instability is quenched by advection. The non-resonant instability that leads to turbulent spectrum $S\left(k_{\|}\right) \propto 1 / k_{\|}^{2}$ acts at larger distances from the shock front than the resonant instability. This latter then supersedes the former, leading to a turbulent spectrum at the shock front $S\left(k_{\|}\right) \propto 1 / k_{\|}$. This onedimensional spectrum is related to the three-dimensional spectrum by $S\left(k_{\|}\right) \equiv(2 \pi)^{-2} \int \mathrm{d}^{2} k_{\perp} S_{3 \mathrm{~d}}\left(k_{\|}, k_{\perp}\right)$, and is normalized according to $\int \mathrm{d} k_{\|} S\left(k_{\|}\right)=2 \pi \delta B^{2} / B_{\infty}^{2}$, where $\delta B$ and $B_{\infty}$ denote respectively the turbulent component and the ISM magnetic field.

On a timescale that is shorter than the instability growth time and the advection time, energy is distributed by resonant three-wave interactions in the perpendicular direction $k_{\perp}$. This redistribution leads to anisotropic three-dimensional turbulent spectra $k_{\perp} S_{3 \mathrm{~d}} \propto k_{\perp}^{-\alpha} k_{\|}^{-\beta}$, with $\beta=1$ as before, and $\alpha=$ $(7-2 \beta) / 3=5 / 3$ if the energy transfer rate of the cascade is a constant independent of scale (Goldreich \& Sridhar 1995; Galtier et al. 2000, 2005). This also implies a relationship between perpendicular and longitudinal wavenumbers: $k_{\|} L_{\max } \propto$ $\left(k_{\perp} L_{\max }\right)^{2 / 3}$, where $L_{\max }$ is the maximum turbulence scale. These results have been found in agreement with recent numerical simulations (Cho \& Vishniac 2000; Maron \& Goldreich 2001).

It has also been shown in Paper I that interactions between two Alfvén and one slow magnetosonic waves provide efficient transfer of energy along the longitudinal direction, which does not arise when only interactions between three Alfvén waves are considered. This allows to maintain the backward parallel spectrum close to the level of the amplified forward spectrum. It has also been pointed out that the non-resonant instability induces a left-right symmetry breaking which provide suitable ground for further amplification by a dynamo. This aspect however has not been studied in detail and is left for further work.

The turbulence that is generated by cosmic ray interactions is compressed along the shock normal at shock crossing. In particular, the perpendicular magnetic field components are amplified by the shock compression ratio $r$ while the longitudinal component remains unchanged. Correspondingly, the turbulent modes wavenumbers $k_{\|}$are increased by the same factor $r$, which corresponds to a similar decrease of the turbulence coherence length in that direction, while perpendicular modes are unchanged. The downstream turbulence is thus even more anisotropic. In the following, we discuss the implications of these turbulent spectra with respect to cosmic ray transport at the shock front, hence with respect to Fermi acceleration.

\section{Diffusion coefficients}

The diffusion coefficients due to resonant pitch angle scattering of cosmic rays with turbulent modes can only be derived analytically in the limit of weak turbulence (quasi-linear theory, Jokipii 1966). Since one expects a high turbulence level in the vicinity of the shock front, the diffusion coefficients have to be computed numerically using Monte-Carlo techniques as described in Casse et al. (2002). The main results of this paper are that the pitch angle frequency follows the same scaling as the quasi-linear theory (hereafter QLT) namely $v_{\mathrm{S}} / \omega_{\mathrm{L}} \sim \eta \rho^{\beta-1}$ for $\rho<1$, where $\beta$ is the index of the turbulence spectrum, $\nu_{\mathrm{s}}=1 / \tau_{\mathrm{s}}$ is the scattering frequency (and $\tau_{\mathrm{s}}$ the scattering time), and $\omega_{\mathrm{L}}$ the Larmor frequency. For $\rho>1$ the ratio $v_{\mathrm{s}} / \omega_{\mathrm{L}}$ decays as $1 / \rho$. The reduced rigidity is defined as:

$\rho \equiv \frac{2 \pi r_{\mathrm{L}}}{L_{\max }}=k_{\min } r_{\mathrm{L}}$ 
with $L_{\max }$ the maximum length of the turbulence and $k_{\min }$ the associated minimum wavenumber. Thus, the parallel diffusion coefficient $D_{\|}=(1 / 3) v^{2} \tau_{\mathrm{s}} \propto \epsilon^{2-\beta}$ for small $\rho(\epsilon$ is the particle energy) and $\propto \epsilon^{2}$ for large $\rho, v \simeq c$ is the cosmic-ray velocity. The transverse diffusion coefficient $D_{\perp}$ has an unusual scaling which neither corresponds to that from quasilinear theory or to the Bohm coefficient, as it is rather controlled by field line chaos, leading to $D_{\perp}=\eta^{2+\varepsilon} D_{\|}$(with $\varepsilon \sim 0.3$ ). In numerical experiments, there is no observed Bohm scaling, only a Bohm maximum for the scattering frequency when $\rho \sim 0,1$ and $\eta \sim 1$.

These scaling laws are modified when anisotropic turbulence is generated, as will be seen in the next section. We have performed new numerical simulations of particle diffusion in turbulent magnetic fields, paying particular attention to the anisotropic nature of the turbulence expected in the vicinity of the shock front. We have decomposed the three-dimensional turbulence on separate discrete grids of perpendicular and parallel wavenumbers. This and the required number of Monte-Carlo runs result in rather long computation times. We have also derived the diffusion coefficients using the quasi-linear theory perturbative methods and used these to interpret the numerical diffusion coefficients, as discussed below.

Assuming the existence of a mean field component $\langle B\rangle$, one defines the pitch angle diffusion frequency $v_{\mathrm{s}}=\tau_{\mathrm{s}}^{-1}=\left\langle\Delta \alpha^{2}\right\rangle / \Delta t$, where $\alpha$ is the pitch angle with respect to the mean field $\langle\boldsymbol{B}\rangle$ and $\Delta \alpha$ the random jump of $\alpha$ during $\Delta t$, with $\tau_{\mathrm{c}} \ll \Delta t \ll \tau_{\mathrm{s}}$. The correlation time $\tau_{\mathrm{c}}$ is of order the Larmor time $\tau_{\mathrm{L}}$ and the latter strong inequalities hold only in weak turbulence regime or in strong turbulence regime for $\rho \ll 1$.

The function $g \equiv v_{\mathrm{s}} / \omega_{\mathrm{L}}$ can be expressed in terms of the three-dimensional turbulent spectrum (see Casse et al. 2002; and Appendix A) as:

$g=\int \frac{\mathrm{d}^{3} k}{(2 \pi)^{3}} S_{3 \mathrm{~d}}\left(k_{\|}, k_{\perp}\right) R(\boldsymbol{k}, \boldsymbol{p})$,

where $\Delta \boldsymbol{x}(\tau)$ and $\omega(k)$ stand for the variation of the unperturbed particle trajectory within a timescale $\tau$ and for the mode pulsation respectively. $R(\boldsymbol{k}, \boldsymbol{p})$ is the resonance function defined by:

$R(\boldsymbol{k}, \boldsymbol{p}) \equiv \int_{0}^{\infty} \mathrm{d}\left(\omega_{\mathrm{L}} \tau\right) \mathrm{e}^{[\mathrm{i} k \cdot \Delta \boldsymbol{x}-\mathrm{i} \omega(k) \cdot \tau]} \cos \left(\omega_{\mathrm{L}} \tau\right)$.

A detailed derivation of the function $g$ including estimates in the strong turbulence regime can be found in the appendix of Casse et al. (2002). The Landau-synchrotron resonances in Eq. (3) are obtained by inserting the gyro-motion, and we get

$$
\begin{aligned}
& R(\boldsymbol{k}, \boldsymbol{p})=\sum_{n=-\infty}^{+\infty} J_{n}^{2}\left(k_{\perp} r_{\mathrm{L}} \sin \alpha\right) \\
& \quad \times\left\{\delta\left[k_{\|} r_{\mathrm{L}} \mu-(n+1)\right]+\delta\left[k_{\|} r_{\mathrm{L}} \mu+(n-1)\right]\right\} .
\end{aligned}
$$

The expression obtained for linearly polarized Alfvén waves in Eq. (4) is identical to other derivations (Schlickeiser 2002; Yan \& Lazarian 2002).

\subsection{Upstream diffusion coefficients}

\subsubsection{Modified weak turbulence spectrum}

We start with a model of weak MHD turbulence (Galtier et al. 2000) upstream modified by the streaming instability of cosmicrays. In that case (see Paper I), the 3D turbulence spectrum reads:

$S_{3 \mathrm{~d}}\left(k_{\|}, k_{\perp}\right)=S_{0} u_{\|}^{-1} u_{\perp}^{-3}$,

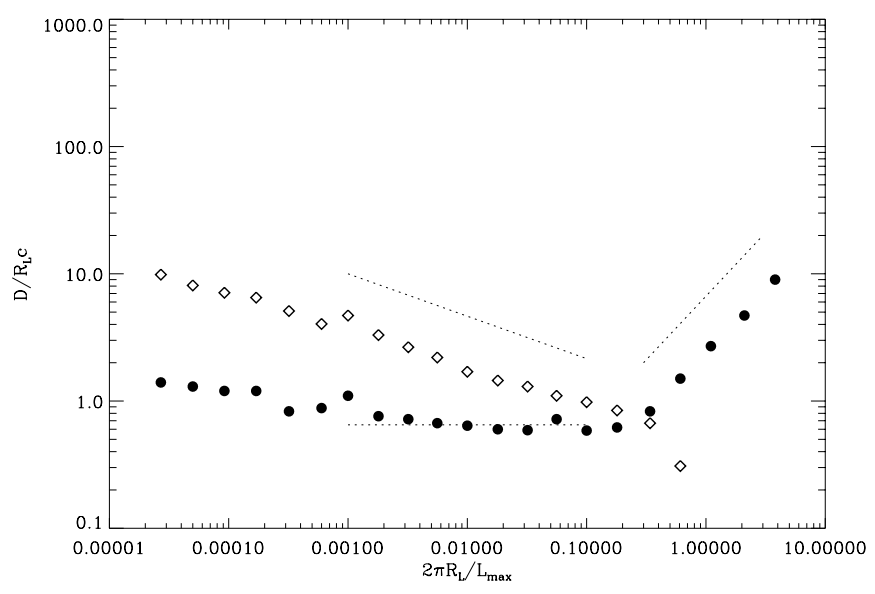

Fig. 1. Parallel (solid dots) and perpendicular (diamonds) diffusion coefficients in units of $r_{\mathrm{L}} c$ as a function of rigidity $2 \pi r_{\mathrm{L}} / L_{\max }, L_{\max }$ denoting the largest scale of the turbulence. The turbulence level is $\eta=0.95$ and the turbulent spectrum $k_{\perp} S_{3 \mathrm{~d}} \propto k_{\perp}^{-5 / 3} k_{\|}^{-1}$. The Bohm diffusion regime is clearly apparent for the parallel diffusion coefficient. The dotted lines have slope $-1 / 3,0$ and 1 . On can see a slight offset in the ordinates of the data points between the two regimes $\rho<10^{-3}$ and $\rho>10^{-3}$; this offset is a numerical artifact, see text for details.

where $u_{\|}=k_{\|} \ell_{\|}$and $u_{\perp}=k_{\perp} \ell_{\perp}$. Out of generality we leave the parallel $\ell_{\|}$and perpendicular $\ell_{\perp}$ magnetic field maximal lengths unspecified; to make contact with previous notations, in isotropic turbulence they would correspond to the maximal length $L_{\max }$ introduced earlier. For a given Larmor radius $r_{\mathrm{L}}$ we have thus two rigidities $\rho_{\|}$and $\rho_{\perp}$.

Since we are mostly interested in the scaling of the diffusion coefficients with rigidity or Larmor radius, we drop the numerical prefactors for convenience. Inserting Eqs. (5) into (4) we find

$g \propto \sum_{n}|n \pm 1| \int_{1}^{\infty} \mathrm{d} u_{\perp}\left[\frac{J_{n}\left(u_{\perp} \rho_{\perp}\right)}{u_{\perp}}\right]^{2}$.

Only the harmonic $n=0$ contributes to the integral significantly. The function $g$ is then found to be constant for $r_{L} \ll \ell_{\perp}$, i.e. the pitch-angle scattering time is proportional to the particle energy. In other words, a turbulence spectrum $S \propto 1 / k_{\|}$as generated by cosmic-ray interactions in the shock precursor leads to Bohm diffusion with $D_{\|} \sim r_{\mathrm{L}} c$.

This result agrees quite well with the numerical computation of the diffusion coefficient in the high turbulence level limit, shown in Fig. 1 for $\eta=0.95$, where $\eta \equiv \delta B^{2} /\left(\delta B^{2}+\langle B\rangle^{2}\right)$. As the turbulence level decreases, the scaling remains the same but the numerical prefactor for the diffusion coefficients increases, see also Casse et al. (2002). This figure reveals a slight offset in the ordinates of the data points between the two regimes $\rho<10^{-3}$ and $\rho>10^{-3}$, which is a numerical artifact. In order to obtain data points down to $\rho \sim 10^{-5}$ when only a limited dynamic range in wavenumber is allowed by computing limitations, we have conducted two separate simulations, one for each rigidity range, and plotted all results together. Data points at $\rho<10^{-3}$ have been obtained from a simulation with the full turbulence spectrum covering five orders of magnitude, i.e. $k_{\max } / k_{\min }=10^{5}$; the simulation for $\rho>10^{-3}$ has a turbulence spectrum that is cut-off at high wavenumbers but with the same $k_{\min }$ as the previous simulation, and $k_{\max } / k_{\min }=10^{3}$. All rigidities have resonant modes with which to interact and computational time is saved by removing the high wavenumber modes (small spatial scales as compared to the Larmor radius) that have a negligible impact on the particle trajectory. This produces a 
slight offset in the diffusion coefficient, which are thus measured to $\sim 10 \%$; however, the trend of a constant $D_{\|} / r_{\mathrm{L}} c$ is clearly seen in both regimes $\rho<10^{-3}$ and $\rho>10^{-3}$.

Note also that, in agreement with the above calculation, the scaling of $D_{\|}$is insensitive to the slope of $S_{3 \mathrm{~d}}$ in the perpendicular direction $k_{\perp}$. On contrary, the perpendicular coefficient is sensitive to the perpendicular cascade, $D_{\perp} \propto r_{\mathrm{L}}^{1 / 3}$.

\subsubsection{Modified Goldreich-Sridhar spectrum}

The second turbulence model we shall consider is the Goldreich-Sridhar model of strong MHD turbulence (Goldreich \& Sridhar 1995) modified by the streaming instability (see Paper I). The three-dimensional turbulence spectrum then reads:

$S_{3 \mathrm{~d}}\left(k_{\|}, k_{\perp}\right)=S_{0} u_{\|}^{-1} u_{\perp}^{-8 / 3} f\left(\frac{u_{\|}}{u_{\perp}^{2 / 3}}-1\right)$,

where $f(x)$ is a smooth function that peaks around $x=0$ and vanishes at infinity. If we approximate $f$ with a Dirac distribution and insert Eqs. (6) into (4), we obtain $g \propto \omega_{\mathrm{L}}^{-1}$ attenuated by $J_{n}^{2}\left(a_{n}\right)$ with argument $a_{n} \equiv\left(\rho_{\|} /|n \pm 1|\right)^{-3 / 2} \rho_{\perp}$. Since the resonance condition corresponds to $u_{\|} \sim 1 / \rho_{\|}$, the GolreichShridar selection for the transverse modes leads to $u_{\perp} \sim u_{\|}^{3 / 2} \sim$ $\rho_{\|}^{-3 / 2} \gg 1$, hence for short transverse wavelengths. Furthermore, for $\rho_{\|} \sim \rho_{\perp} \ll 1$, the argument $a_{n} \gg 1$ and the large argument limit of Bessel functions gives:

$g \sim \eta \rho_{\|}^{5 / 2} / \rho_{\perp}$

We obtain identical results if $f$ is approximated by a step function or an exponential form.

In this anisotropic turbulence, whose spectrum is constrained by the relation between $k_{\|}$and $k_{\perp}$, the parallel diffusion coefficient $D_{\|}$shows a different scaling than in unconstrained turbulence. On a formal level, this difference is related to the fact that the relation between $k_{\|}$and $k_{\perp}$ no longer permits to treat perpendicular and parallel wavenumbers separately in the integrals. The QLT result that we obtained in Eq. (7) indicates that the perpendicular spectrum at short wavelengths influences the pitch angle scattering frequency.

In the numerical calculation whose results are presented in Fig. 2, we have approximated the function $f(x)$ with a step function that vanishes outside of the interval $[-1,+1]$ and used, as before, a turbulence level $\eta=0.95$. We find that the parallel diffusion coefficient no longer respects the Bohm scaling, but rather follows the perpendicular coefficient and scales as in Kolmogorov diffusion, $D_{\|} \propto \rho^{1 / 3}$. This behavior, which does not agree with the above quasi-linear estimate Eq. (7), may be understood in the following way.

The quasi-linear calculation indicates that diffusion in this contrainted turbulence cannot be induced by resonant interactions in the longitudinal direction $k_{\|} r_{\mathrm{L}} \sim 1$. Indeed, if this resonance condition is satisfied, the Goldreich-Shridar prescription $k_{\|} l_{\|} \propto k_{\perp}^{2 / 3} l_{\perp}^{2 / 3}$ indicates that $k_{\perp} r_{\mathrm{L}} \gg 1$ and this large argument of the Bessel function in Eq. (4) then kills the contribution of these interactions in $g$. Since the parallel and perpendicular diffusion coefficients are found to satisfy $D \propto r_{\mathrm{L}}^{1 / 3}$, as was observed in unconstrained turbulence for $D_{\perp}$, the numerical simulation rather indicates that diffusion is now controled by perpendicular wavenumbers, i.e. $k_{\perp} r_{\mathrm{L}} \sim 1$. If indeed those interactions dominate and the correlation time remains of the order of the Larmor

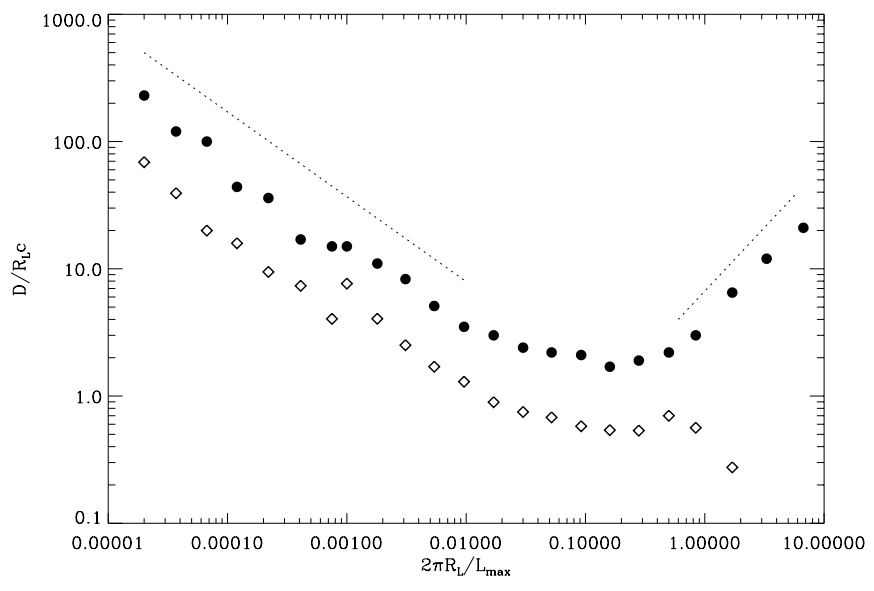

Fig. 2. Parallel (solid dots) and perpendicular (diamonds) diffusion coefficients in units of $r_{\mathrm{L}} c$ as a function of rigidity $2 \pi r_{\mathrm{L}} / L_{\max }$. The turbulence level is $\eta=0.95$ and the turbulent spectrum $k_{\perp} S_{3 \mathrm{~d}} \propto k_{\perp}^{-5 / 3} k_{\|}^{-1}$ with the constraint that $\left(k_{\perp} L_{\max }\right)^{2 / 3}>k_{\|} L_{\max }$. The dotted lines have slope $-2 / 3$ and 1 , and indicate that the diffusion coefficients at low rigidities scale as $r_{\mathrm{L}}^{1 / 3}$ as in isotropic Kolmogorov turbulence.

time, the discussion in Appendix A shows that the observed behavior can be reproduced. In this description, pitch angle scattering in the parallel direction takes place when the particle jumps from one magnetic field line to another and the orientation of these field lines differ from one another. Diffusion along the parallel direction is then controled by perpendicular diffusion, as is apparent in the results of the numerical simulations shown in Fig. 2.

Following the discussion of Appendix A, we finally obtain:

$\frac{v_{\mathrm{S}}}{\omega_{\mathrm{L}}} \sim \eta \rho_{\perp}^{2 / 3}$

It is interesting to note that the exponent does not depend on the turbulence spectral indices $\alpha$ and $\beta$ in a first approximation (see Appendix A).

For $\rho_{\|}>1$, the arguments of the Bessel function are small and we find again the same result as in the isotropic case, namely a parallel diffusion coefficient increasing like $\rho_{\|}^{2}$. Interestingly, the above diffusion coefficients should be expected to apply to propagation in the interstellar medium as well if the streaming instability of CR along the mean magnetic field is the dominant source of turbulence generation. A detailed investigation of this issue is postponed to a future work.

\subsection{Downstream diffusion coefficients}

Downstream of the shock the parallel and perpendicular coherence lengths are different: they are related to the coherence length upstream $L_{\max }$ by $\ell_{\perp}=L_{\max }$ and $\ell_{\|}=L_{\max } / r$. The turbulence transforms in a non-trivial way, since the magnetic field modes components that are perpendicular to the shock normal are amplified by $r$, and these modes may be associated with wavenumbers that are neither completely parallel nor completely perpendicular. The overall turbulence magnetic field is nevertheless amplified by $R=\sqrt{\left(1+2 r^{2}\right) / 3}$ after statistical averaging, while the coherent magnetic field, which was assumed parallel, is left unmodified. Hence the turbulence amplitude $\eta$ changes from upstream to downstream by the above amplification factor $R$ if $\eta \ll 1$, or remains the same if $\eta \sim 1$.

The effective value of the compression ratio explored by the particles depends on their rigidity in the case of a strongly 


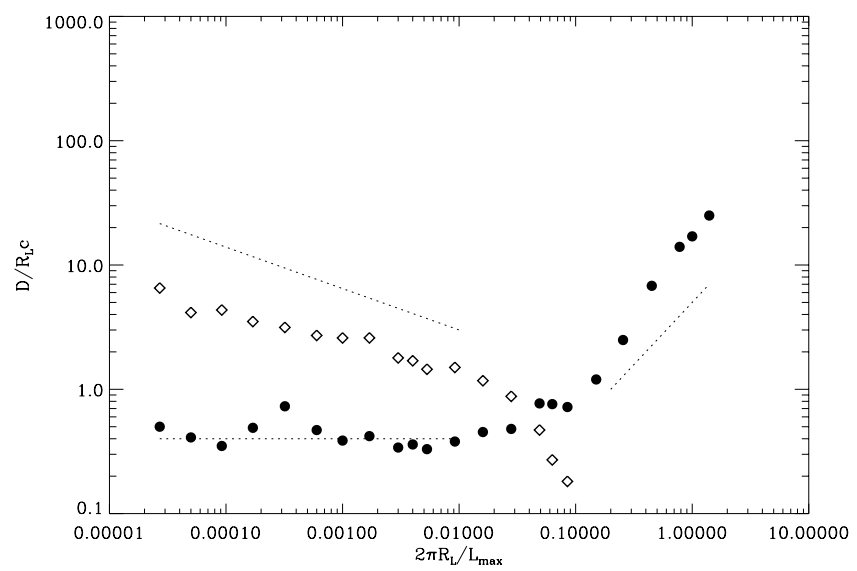

Fig. 3. Same as Fig. 1, i.e. a turbulence spectrum $k_{\perp} S_{3 \mathrm{~d}} \propto k_{\perp}^{-5 / 3} k_{\|}^{-1}$ and $\eta=0.95$ but accounting for the compression of the turbulence through the shock.

modified shock. Only the CRs of highest energy should explore the total compression ratio, $r_{\text {tot }}$ while low-energy particles explore the much lower compression ratio of the subshock, $r_{\text {sub }}$, and the electrons of highest energy explore a region across the shock with an intermediate effective compression ratio $r_{\mathrm{el}}$. The fluid quantities (density, velocity, thermal pressure, tangential magnetic field) are all subject to a jump dependent on $r_{\text {sub }}$ at the shock precursor. For instance the magnetic field compression $\mathrm{R}$ ratio should be expressed in terms of $r_{\text {sub }}$ instead of $r$. Typical values are $r_{\text {sub }} \simeq 2-3$ and $r_{\text {tot }} \simeq 7-10$ (Berezhko et al. 1996; Bykov 2004). However, in Sect. 6 we will show that a consistent modelling of young $\mathrm{SNr}$ with a strong magnetic field amplification by the streaming of CR should include both the $\mathrm{CR}$ backreaction on the shock structure and the $\mathrm{CR}$ distribution steepening produced while generating the turbulence. The last effect will tend to diminish the CR pressure and to reduce the non-linear shock modification. One may expect in that case $r_{\text {sub }}$ not to be strongly different from the the test particle case.

Mainly for the previous reason and also as they would only complicate the formal treatment with no significant change in the results we shall not go into such details in this paper. (Accounting for a different compression ratio $r_{\text {sub }}$ and $r_{\mathrm{el}}$ would lead to a variation of the order of $30 \%$ for the deduced value of $E_{\mathrm{p} \text {,max }}$ as already stressed in Parizot et al. 2006.) In any case, we found that a situation with explicitly different values of the compression ratio for nuclei and electrons in the case of a strongly modified shock results in an intermediate solution between the test-particle case (no precursor nor sub-shock, and $r=4$ ) and the pure $r=10$ case expected in strongly modified shocks (see Bykov 2004). We shall thus confine the study below to such idealised cases, using only one ("universal") compression ratio $r$ for all particles and the fluid quantities.

The transport properties differ in compressed and uncompressed turbulence, see for instance Lemoine \& Revenu (2005) for the case of ultra-relativistic shocks. In the following Figs. 3, 4 we show the diffusion coefficients of particles propagating in compressed turbulence (with $r=4$ ) for the same cases of turbulence spectra as shown in Figs. 1, 2.

The comparison of these figures reveals that the main effect of compressed turbulence comes through the reduction of the parallel coherence length: in compressed turbulence, the scaling of $D_{\|}$with respect to rigidity $2 \pi r_{\mathrm{L}} / L_{\max }$ remains the same as in uncompressed turbulence, but the curve is shifted to lower values of the rigidity. Hence if one plotted $D_{\|}$versus $r \times 2 \pi r_{\mathrm{L}} / L_{\max }$, most of the discrepancy would disappear. One also observes that

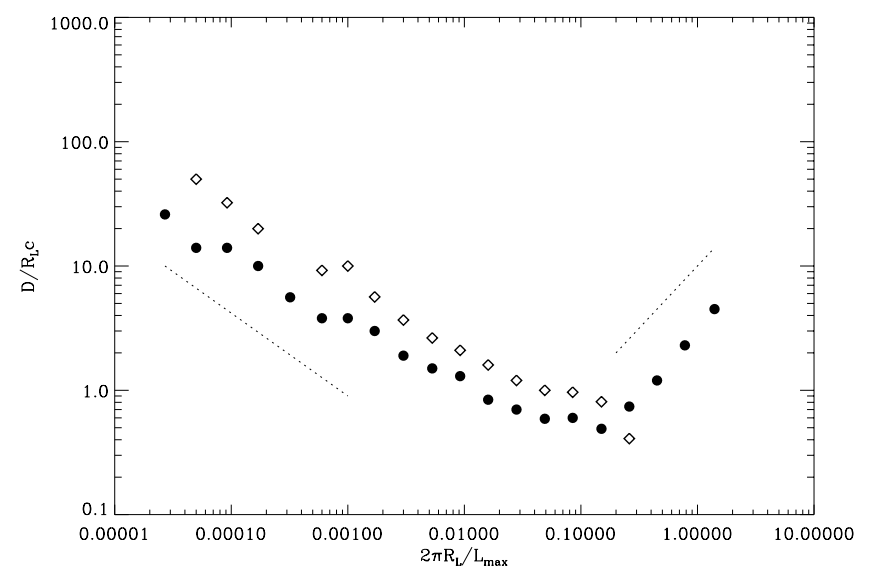

Fig. 4. Same as Fig. 2, i.e. assuming that $\left(k_{\perp} L_{\max }\right)^{2 / 3}>k_{\|} L_{\max }$, and accounting for the compression of the turbulence through the shock.

the parallel, and to some a smaller extent the perpendicular diffusion coefficients are decreased to a lower value. The difference in turbulence level between downstream and upstream is probably too small to account for this difference, since $\eta=0.95$ upstream and $\eta=0.98$ downstream. In the case of a modified GoldreichSridhar spectrum, a reduction factor of $\simeq 4^{2 / 3}$ of the maximum turbulence length can explain this difference.

Another consequence of magnetic field amplification and compression at the shock front is that the ratio of the downstream Alfvén velocity $V_{\text {Ad }}$ to the downstream fluid velocity $V_{\mathrm{d}}$ is unlikely to be much smaller than unity as in the upstream medium far from the shock front. In detail, the downstream Alfvénic Mach number $\mathcal{M}_{\mathrm{Ad}} \equiv V_{\mathrm{d}} / V_{\mathrm{Ad}}$ is expressed in terms of the upstream Alfvénic Mach number $\mathcal{M}_{\mathrm{Au}}$ as:

$\mathcal{M}_{\mathrm{Ad}} \simeq r^{-3 / 2} \mathcal{M}_{\mathrm{Au}}$

due to compression of the magnetic field and density by $\simeq r$. With $r \simeq 4$ and $\mathcal{M}_{\mathrm{Au}} \sim 10$ (see Eq. (18) below), one finds $V_{\mathrm{Ad}} \sim V_{\mathrm{d}}$. In this case, second-order Fermi acceleration should be taken into account during the Fermi cycle. The stochastic momentum diffusion coefficient is easily expressed in the relativistic limit as

$D_{p p}=\frac{1}{3} p^{2}\left(\frac{V_{\mathrm{A}}}{c}\right)^{2} v_{\mathrm{s}}=\frac{1}{9} p^{2} \frac{V_{\mathrm{A}}^{2}}{D_{\|}}$,

and $p=\epsilon / c$ is the particle momentum. The Fermi second order acceleration timescale, $t_{\mathrm{FII}}=\left(D_{p p} / p^{2}\right)^{-1} \propto D_{\|}$is then controled by the downstream stochastic diffusion coefficient. This contribution of stochastic reacceleration downstream is discussed in Sect. 5 .

\section{Revised Fermi cycle in shocks with magnetic field amplification}

Each relativistic particle streaming upstream of the shock front generates shear Alfvén waves. Accounting for the possibility of strong turbulence, the energy loss in generating the waves is

$P(\epsilon)=-\frac{1}{3} \frac{v^{2}}{c^{2}} V\left(\frac{\partial}{\partial x} \log f\right) \epsilon$,

(see Appendix B), $V$ the velocity of the scattering centers upstream is close to the local Alfvén velocity $V_{\mathrm{Au}}$.

This general expression can be specified upstream where the distribution function is governed by the following transport equation, assuming a stationary state:

$-V_{\mathrm{u}} \frac{\partial}{\partial x} f+\frac{\partial}{\partial x} D_{\mathrm{u}} \frac{\partial}{\partial x} f=0$, 
The power loss, in most of the upstream region where $f \gg f_{\mathrm{u}}$, is then given by

$P(\epsilon)=-\frac{1}{3} \frac{v^{2}}{c^{2}} V \frac{V_{\mathrm{u}}}{D_{\mathrm{u}}} \epsilon$.

The average duration of a half Fermi cycle upstream is $4 D_{\mathrm{u}} /\left(V_{\mathrm{u}} v\right)$. A particle that undergoes such wandering and thus contributes to turbulence generation spends an average amount of energy $-\frac{4}{3} \frac{V v}{c^{2}}$. The energy gain per Fermi cycle $G \equiv 1+\xi$ is thus diminished for two reasons, first by the fact that the particles experience a reduced velocity difference between scattering centers $V_{\mathrm{u}}-V_{\mathrm{d}}-V$ and moreover loose energy by exciting turbulence; then

$\xi=\frac{4}{3} \frac{V_{\mathrm{u}}-V_{\mathrm{d}}}{v}-\frac{4}{3}\left(\frac{V}{v}+\frac{V v}{c^{2}}\right)$.

Since the escape probability per cycle is unaffected, $P_{\mathrm{esc}}=$ $4 V_{\mathrm{d}} / v$, the amplification of upstream turbulence and the concomittant reduction of the energy gain steepen the accelerated spectrum. The spectral index is given by (Bell 1978):

$s=1+\frac{\log 1 / P_{\mathrm{ret}}}{\log G} \simeq 1+\frac{P_{\mathrm{esc}}}{\xi} \simeq 1+\frac{3}{r-1-2 \frac{V}{V_{\mathrm{d}}}}$,

or

$s \simeq 1+\frac{3}{(r-1)} \times\left[1+2 \frac{r}{(r-1)} \frac{V_{\mathrm{Au}}}{V_{\mathrm{sh}}}\right]$,

or again

$s \simeq 2+\frac{8}{3} \frac{V_{\mathrm{Au}}}{V_{\mathrm{sh}}}$

for a compression ratio $r=4$. From Eq. (15), we can see that the effect of wave generation upstream on the CR spectrum is important once the amplitude of the fluctuations $\delta B$ becomes much larger than the ISM magnetic field. Let us quantify this remark. The index can be expressed in terms of the inverse of the turbulent (upstream) Alfvén Mach number

$M_{\mathrm{A}}=\frac{V_{\mathrm{sh}}}{V_{\mathrm{au}}} \simeq 14.3\left(\frac{B}{100 \mu \mathrm{G}}\right)^{-1}\left(\frac{n}{1 \mathrm{~cm}^{-3}}\right)^{1 / 2} \frac{V_{\mathrm{sh}}}{10^{-2} c}$.

Instead of an index of 2 for strong shocks, we get for a $\mathrm{SNr}$ shock $V_{\text {sh }}=5000 \mathrm{~km} \mathrm{~s}^{-1}$, with an amplified upstream magnetic field of $\simeq 100 \mu \mathrm{G}$ (Berezhko \& Völk 2004; Parizot et al. 2006) in a hot ISM medium $n_{\mathrm{HIM}} \simeq 4 \times 10^{-3} \mathrm{~cm}^{-3}, s=2+1.5$ and in a warm ISM medium $n_{\text {WIM }} \simeq 10^{-1} \mathrm{~cm}^{-3}, s=2+0.35$ respectively. The index appears to be strongly modified especially in the hot phase.

The compression ratio can also be changed with a correction of order $1 / \mathcal{M}_{\mathrm{Au}}$ by the modification of the shock by both $\mathrm{CR}$-pressure gradient and wave-pressure gradient. Note that the thermal plasma pressure also increases in the precursor because the magnetic energy cascade towards dissipation scale where cyclotron absorption takes place. These effects tend to diminish the compression ratio. Using Eq. (18) for $r=10$ the spectral index $s \simeq 1.65$ in the warm ISM is found to be slightly harder than the solution obtained in modified shock simulations $(s \simeq 1.5$, Ellison et al. 2004). All these effects are still to be integrated in numerical simulations of CR modified shocks including kinetics and the effect of a strong Alfvén production upstream (see Jones 1994, for a discussion in the three fluid approximation).

As already mentioned, the generation of a highly amplified and compressed magnetic field enhances the Alfvén velocity compared to far upstream in such a way that the Fermi second ordrer process may become important. However, if only forward waves are generated, the Fermi second order process does not work. Assuming that the energy is distributed evenly between forward and backward waves, one can estimate the mean energy gain through second order acceleration using Eq. (10):

$g_{\mathrm{FII}} \simeq \frac{4}{3} \frac{v V_{\mathrm{d}}}{c^{2}}\left[\left(\frac{V_{\mathrm{Ad}}}{V_{\mathrm{d}}}\right)^{2} \frac{1}{p^{3} v_{\mathrm{sd}}} \frac{\partial\left(p^{4} v_{\mathrm{sd}}\right)}{\partial p}\right]$

The overall particle energy gain/loss during one cycle transforms to:

$G_{\mathrm{tot}}=1+\frac{4}{3} \frac{V_{\mathrm{u}}-V_{\mathrm{d}}}{v}-\frac{4}{3}\left(\frac{V_{\mathrm{Au}}}{v}+\frac{V_{\mathrm{Au}} v}{c^{2}}\right)+g_{\mathrm{FII}}$.

If we note $g_{\mathrm{FI}}=4 / 3\left(V_{\mathrm{u}}-V_{\mathrm{d}}\right) / v$ the mean energy gain corresponding to the first Fermi process, then the use of Eq. (19) gives:

$\frac{g_{\mathrm{FII}}}{g_{\mathrm{FI}}} \simeq \frac{3-\kappa}{r-1}\left(\frac{V_{\mathrm{ad}}}{V_{\mathrm{d}}}\right)^{2}$,

where $\kappa$ is defined by the energy (rigidity) dependence of the diffusion coefficient: $D_{\|} \propto D_{\text {Bohm }} \rho^{\kappa} \propto \rho^{\kappa+1}$, with $D_{\text {Bohm }} \equiv r_{\mathrm{L}} c$. If the anisotropy of the turbulence is not constrained by a relation of the form $k_{\|} \propto k_{\perp}^{2 / 3}$, as discussed in Sect. 4.1.1, then $\kappa=1-\beta$, with $\beta$ the index of the turbulence power spectrum as a function of $k_{\|}$. In particular, $\beta=1$ implies $\kappa=0$, hence a Bohm diffusion coefficient.

Usually, magnetic field strength up to $500 \mu \mathrm{G}$ are derived in young $\mathrm{SNr}$ producing non-thermal X-ray radiation. The previous equation implies that the second order process can become comparable or even dominate the regular Fermi process for ISM densities lower than a few tens (corresponding to the hot ISM phase) for such high magnetic field strengths. The stochastic process has then to be incorporated in a self-consistent way in the Fermi cycle in such cases. However, in order to do so one has to take into account the likely anisotropy of the turbulence between backward and forward waves and evaluate its effect on the efficiency of the second order Fermi process. This investigation remains beyond the scope of this paper and is left for future work.

\section{Discussion: cosmic-ray acceleration in $\mathrm{SNr}$}

In this section, we aim at deriving the maximum $\mathrm{CR}$ energies produced by the diffusive shock acceleration processes in an anisotropic turbulence. We limit our calculations to the young SNr showing thin X-ray filaments as observed by the satellites Chandra and XMM-Newton (see Ballet 2005). We postpone to a future work the derivation of the overall source CR spectrum produced by $\mathrm{SNr}$ during their whole evolution phases as discussed recently in a similar context by Ptuskin \& Zirakshvilli (2005).

In Sect. 4, we have derived the general form of the diffusion coefficient up- and down-stream. Expressed in terms of the Bohm diffusion coefficient $D_{\mathrm{Bohm}} \equiv r_{\mathrm{L}} c$, we get:

$$
\begin{aligned}
D(\rho) & =k D_{\text {Bohm }}\left(\frac{\rho}{\rho_{p}}\right)^{\alpha}, \text { for } \rho \leq \rho_{\mathrm{p}} \\
& =k D_{\text {Bohm }}\left(\frac{\rho}{\rho_{p}}\right)^{2}, \text { for } \rho>\rho_{\mathrm{p}} .
\end{aligned}
$$


From the simulations we infer approximatively $\rho_{\mathrm{pu}} \simeq 0.2$ upstream and $\rho_{\text {pd }} \simeq 0.2 / r$ downstream, $r$ being the shock compression ratio. The normalisation factor $k$ is also different up- and downstream. In the case of a Bohm type turbulence upstream, compressed by a factor $r$ downstream (assumed to be 4 hereafter) we have $k_{\mathrm{u}} \simeq 2$ and $k_{\mathrm{d}} \simeq 4$ respectively (see Figs. 1 and 3 ). In case of a Kolmogorov type turbulence upstream, compressed by a factor $r$ downstream, $k_{\mathrm{u}} \simeq 6$ and $k_{\mathrm{d}} \simeq 6 / 4^{2 / 3} \sim 2.4$ (see Figs. 2 and 4).

In order to discuss the particle acceleration process in young $\mathrm{SNr}$ quantitatively, all the lengths are compared to the size of the $\mathrm{SNr}$ and all the timescales to the age of the SNr. The maximum scale of the turbulence up-stream and the particle gyroradius can be expressed in terms of the $\mathrm{SNr}$ shock radius units, namely $L_{\max }=\bar{L}_{\max } \times R_{\mathrm{sh}} r_{\mathrm{L}}=\bar{r}_{\mathrm{L}}(E, B) \times R_{\mathrm{sh}}$ with, $\bar{r}_{\mathrm{L}}=\bar{r}_{0} E_{\mathrm{PeV}} B_{100}^{-1}, E_{\mathrm{PeV}}$ is the CR energy in $\mathrm{PeV}$ and $B_{100}$ is the magnetic field intensity in $100 \mu$ Gauss units. Hereafter, we will restrict our analysis to young $\mathrm{SNr}$ in a free expansion phase for which the forward shock velocity is the largest. This leads to a direct relationship between the $\mathrm{SNr}$ radius and age $R_{\mathrm{sh}} \simeq V_{\mathrm{sh}} \times t_{\mathrm{SNR}}:$

$R_{\mathrm{sh}} \simeq \frac{3}{2} \mathrm{pc} \frac{V_{\mathrm{sh}}}{5000 \mathrm{~km} \mathrm{~s}^{-1}} \times \frac{t_{\mathrm{SNR}}}{300 \mathrm{yr}}$

hence $\bar{r}_{0} \simeq 2 \times 10^{-2}$ for these values. We now derive the explicit values of the maximum cosmic-ray energies.

Note that downstream, the turbulence may also relax towards ISM values over a scale $\ell_{\mathrm{r}}(E)$ (Pohl et al. 2005) producing a diffusion coefficient $D_{\mathrm{d}} \simeq D_{\mathrm{d}}\left(\ell_{\mathrm{r}} \rightarrow \infty\right) \times \exp \left(x(\alpha+1) / 2 \ell_{\mathrm{r}}\right)$, with $D_{\mathrm{d}}\left(\ell_{\mathrm{r}} \rightarrow \infty\right)$ the diffusion coefficient in a uniform medium as before. Relaxation scales $\ell_{\mathrm{r}} \ll R_{\mathrm{sh}}$ have for average effect to increase the particle residence downstream and then the acceleration timescale. It is expected that acceleration through a relaxed turbulence is less efficient in producing high energy CR. A detailed investigation of this process deserves to be pushed further.

Maximum CR energy in young $S N r$ : if the relaxation scale is $\ell_{\mathrm{r}} \sim R_{\mathrm{sh}}$ then the X-ray filaments observed in some young $\mathrm{SNr}$ are limited by the radiative (synchrotron) losses. Several authors (Berezhko et al. 2003; Berezhko \& Völk 2004; Parizot et al. 2006) have derived in a self-consistent manner the downstream magnetic field by comparing the observed filament size $\Delta R_{\text {rim }}$ with combined advection and diffusive lengths explored by the relativistic electrons during their synchrotron loss timescale. The magnetic field strengths obtained are of the order of 300-400 $\mu \mathrm{G}$ in the youngest SNr like Kepler and Tycho and $100 \mu \mathrm{G}$ in older SNr like SN1006. These estimates however have assumed an isotropic turbulence up- and downstream.

As discussed in the introduction, the anisotropy considered here has two main effects. Firstly, if the Goldreich-Sridhar scaling does apply then diffusion has a Kolmogorov scaling even if the streaming instability tends to produce a spectrum $\propto k_{\|}^{-1}$. Secondly, this turbulence spectrum is transmitted downstream and the scales parallel to the shock normal (in a quasi-parallel shock configuration) are compressed by a factor close to $r$. We shall account for both these effects in the following estimates.

We first derive the magnetic field downstream using the size of the X-ray filaments (see Berezhko \& Völk 2004; and Parizot et al. 2006). The electron particle distribution $f(x)$ verifies $V_{\mathrm{d}}(\partial f / \partial x)=D \partial^{2} f / \partial x^{2}-f / \tau_{\text {syn }}$. In this equation, catastrophic losses for $\mathrm{TeV}$ electrons have been assumed. The solution is of the form $f(x) \propto \exp \left(-x / \Delta R_{\text {rim }}\right)$. The magnetic field downstream then follows from:

$\left(\frac{D}{\Delta R_{\mathrm{rim}}}\right)^{2}+\frac{V_{\mathrm{d}}}{\Delta R_{\mathrm{rim}}}=\frac{1}{\tau_{\mathrm{syn}}}$

The synchrotron loss time $\tau_{\text {syn }} \simeq(95 \mathrm{yr}) B_{\mathrm{d} 100}^{-3 / 2} E_{\mathrm{obs}-\mathrm{keV}}^{-1 / 2}$, where $E_{\text {obs-keV }}$ is the photon energy in $\mathrm{keV}$ units at which the X-ray filaments are observed and $B_{\mathrm{d} 100}$ is the downstream magnetic field in units of $100 \mu \mathrm{G}$. Once the dependence of the diffusion coefficient on particle energy is known, Eq. (24) leads to a one to one relationship between the downstream magnetic field and $\Delta R_{\text {rim }}=\Delta \bar{R}_{\text {rim }} R_{\mathrm{sh}}$. The ratio of the upstream to downstream magnetic field is approximately $1.2 / r$ (Parizot et al. 2006). The maximum cosmic-ray energy $E_{\mathrm{pmax}}$ is then calculated by balancing the diffusive length upstream $D_{\mathrm{u}} / V_{\mathrm{sh}}$ with the shock radius (see Hillas 2005, for a discussion). In order to fix the maximum scale of the turbulence, let us first assume $L_{\max }=r_{\mathrm{L}}\left(E_{\mathrm{pmax}}\right)$.

Bohm regime: in the case where diffusion proceeds according to a Bohm regime upstream, $\kappa=0$. The magnetic field downstream is then derived as $B_{\mathrm{d}} \simeq 400 \mu \mathrm{G}$ for a typical filament size $\Delta \bar{R}_{\text {rim }}=10^{-2}$ and $r=4$. The results are similar for $r=10$ in case of a strongly modified shock. Such high magnetic fields downstream require an efficient amplification in the shock precursor (see Paper I). Using the above argument on upstream CR escape, we find maximum cosmic ray energies $E_{\mathrm{pmax}} \simeq 1.5 \mathrm{PeV}$ for $r=4$ or $0.6 \mathrm{PeV}$ for $r=10$.

Kolmogorov regime: if the upstream turbulence is of the Goldreih-Shridar type, the diffusion is similar to Kolmogorov, $\kappa=-2 / 3$ and Eq. (24) leads to a magnetic field of the order of $B_{\mathrm{d}} \simeq 550 \mu \mathrm{G}$ for filament size $\Delta \bar{R}_{\text {rim }}=10^{-2}$ and $r=4$, $B_{\mathrm{d}} \simeq 400 \mu \mathrm{G}$ for $r=10$. Using the above argument on upstream CR escape, we find maximum cosmic ray energies $E_{\text {pmax }} \simeq 7 \mathrm{PeV}(r=4)$ or $\simeq 2 \mathrm{PeV}(r=10)$.

These estimates suggest that it is possible to accelerate $\mathrm{CR}$ up to the CR knee with magnetic field amplification in the shock precursor for both Bohm and Kolmogorov regimes. This confirms earlier results found for isotropic turbulence (Parizot et al. 2006) even if the typical maximum energies for CR tend to be slightly higher in case of anisotropic compressed turbulence. Another difference with the isotropic turbulence case, is that the Kolmogorov regime can not be ruled-out here; the rejection condition $D \leq D_{\text {Bohm }}$ is not true anymore in anisotropic turbulence. However, it seems still challenging at this SN evolution stage to accelerate the particles up to the CR ankle (see Ptuskin \& Zirakshvilli 2005; Bykov 2001; and Parizot et al. 2004, for alternative scenario).

The above maximum CR energies are probably slightly overestimated since the diffusion coefficient $D \propto \rho^{2}$ at high rigidities and the hypothesis $L_{\max }=r_{\mathrm{L}}\left(E_{\mathrm{pmax}}\right)$ is optimistic. In order to estimate this uncertainty, we use a supplementary relation to fix $L_{\text {max }}$. For instance, the condition $\tau_{\text {acc }}\left(E_{\text {emax }}\right)=\tau_{\text {sync }}\left(E_{\text {emax }}\right)$ at the maximum electron energy provides a one to one relationship between $L_{\max }$ and the magnetic field. For the youngest $\mathrm{SNr}$, the typical synchrotron cut-off frequency is of the order of $1 \mathrm{keV}$. Downstream magnetic fields in both regimes are still of the order of $400-500 \mu \mathrm{G}$. In the Kolmogorov regime, $\bar{L}_{\max } \simeq 10^{-2}$ and the maximum $\mathrm{CR}$ energies cannot lie well beyond $0.3 \mathrm{PeV}$ as a result of the scaling $D \propto \rho^{2}$ at high rigidities.

To summarize, the above estimates give as an order of magnitude for the maximum energy of cosmic 
rays: $E \sim Z \times(0.3-3) \mathrm{PeV}$ in young $\mathrm{SNr}$ showing X-ray filaments if the magnetic field is amplified by the streaming instability.

This paper only considered the free expansion stage of SNr evolution and did not investigate the overall spectrum, expected to be steep beyond the CR knee (Ptuskin \& Zirakshvilli 2005). The maximum CRs energies calculated here are estimates expected once a strong magnetic field amplification is at work at the shock precusor as deduced from X-ray observations from young SNr. Further work should take into account the different $\mathrm{SNr}$ evolution phases either, as well as the different type of SN (core-collapsed or type Ia). A consistent spectrum derivation should account for both upstream wave generation and shock smoothing by the cosmic-ray pressure as well as the second order Fermi acceleration process downstream. Consequently, a detailed reconstruction of the CR spectrum produced by an ensemble of SNr during their evolution is beyond the scope of the paper and will be adressed in future work.

\section{Conclusion}

In this Paper II, we have shown that the diffusion coefficients of cosmic rays can be significantly modified in the precursor of a shock, especially because of the formation of an anisotropic turbulence spectrum.

According to Paper I, the streaming instability shapes the power spectrum of the turbulence according to the scaling $S_{3 \mathrm{~d}} \propto$ $k_{\|}^{-1} k_{\perp}^{-\alpha-1}$, while the transverse index $\alpha$ depends on the modeling of the Alfvén cascade. In the absence of any correlation between parallel and transverse wave numbers, the spectrum factorizes and the diffusion coefficients are insensitive to the transverse distribution. The parallel diffusion coefficient respects a Bohm scaling, $D \propto r_{\mathrm{L}} c$. However, if a correlation à la GoldreichShridar arises, i.e. $k_{\|} \ell_{\|} \sim\left(k_{\perp} \ell_{\perp}\right)^{2 / 3}$, the results differ from the quasi-linear prediction. Numerical simulations indicate that the parallel diffusion coefficient scales with rigidity as in isotropic Kolmogorov turbulence. This behavior is understood if pitch angle scattering is now controled by transverse transport from one field line to another. In any case these results allow to explain the recent X-ray observations young Supernova remnants. The diffusion coefficient for the electrons, in particular, agrees well with the expected size of the filaments.

The energy loss per particle that accompanies the generation of turbulence is calculated. The cosmic-ray energy spectrum is consequently steepened, which supports the suggestion that the index at the source is closer to 2.3 (index obtained in the warm ISM phase) rather than 2. A further steepening due to the diffusive propagation in the interstellar medium would likely leads to an index close to 2.7 as long as the diffusion develops with a Kolmogorov spectrum. Finally the amplification of the magnetic field allows to push the maximal energy cut-off into the "knee" region ( $\mathrm{PeV}$ energies). Consistent future investigations with the cosmic ray data should require the inclusion of the magnetic field amplification by the streaming instability, the shock modification by the cosmic ray pressure and possibly the effect of stochastic Fermi acceleration downstream the shock.

Acknowledgements. The authors thank an anonymous referee for helpful comments. A.M. acknowledges fruitful discussions with J. Ballet, F. Casse and E. Parizot.

\section{Appendix A: Pitch angle diffusion}

The variations of the pitch angle are governed by a simple stochastic equation that stems from the projection of the Lorentz equation along the mean field by taking into account the energy conservation (and thus the conservation of $p$ and $v$ ). This gives:

$\dot{\alpha}=f(t) \equiv \omega_{\mathrm{L}}\left[\cos \phi(t) b_{2}(t)-\sin \phi(t) b_{1}(t)\right]$,

where $\omega_{\mathrm{L}} \equiv Z e \bar{B} / m \gamma c, \phi(t)$ is the gyro-phase, i.e. $\dot{\phi}(t)=\omega_{\mathrm{L}}+$ $O(b)$ and $\boldsymbol{b} \equiv \delta \boldsymbol{B} / \bar{B}$ is the irregularity of the field experienced by the particle along its trajectory. For a stationary process, the pitch angle frequency is thus

$v_{\mathrm{s}} \equiv \frac{\left\langle\Delta \alpha^{2}\right\rangle}{\Delta t}=2 \int_{0}^{\infty}\langle f(\tau) f(0)\rangle \mathrm{d} \tau$,

which can be rewritten as follows:

$v_{\mathrm{s}}=\omega_{\mathrm{L}}^{2} \int_{0}^{\infty}\langle\boldsymbol{b}(\tau) \cdot \boldsymbol{b}(0) \cos \Delta \phi(\tau)\rangle \mathrm{d} \tau$

If the level of turbulence is sufficiently weak, one approximates $\Delta \phi(\tau) \simeq \omega_{\mathrm{L}} \tau$ and $\boldsymbol{b}(\tau)$ is expressed in term of the unperturbed trajectory. This leads to the expression:

$v_{\mathrm{s}}=\omega_{\mathrm{L}} \int \frac{\mathrm{d}^{3} k}{(2 \pi)^{3}} S_{3 \mathrm{~d}}(\boldsymbol{k}) R(\boldsymbol{k}, \boldsymbol{p})$,

where $S_{3 \mathrm{~d}}(\boldsymbol{k})$ is the 3D correlation spectrum of the field irregularities normalized such that its integral equals to the irregularity degree $\eta$ :

$\eta \equiv \frac{\left\langle\delta \boldsymbol{B}^{2}\right\rangle}{\left\langle\boldsymbol{B}^{2}\right\rangle}=\int \frac{\mathrm{d}^{3} k}{(2 \pi)^{3}} S_{3 \mathrm{~d}}(\boldsymbol{k})$,

and the resonance function $R(\boldsymbol{k}, \boldsymbol{p})$ describes the resonant interaction between the particles and the modes:

$R(\boldsymbol{k}, \boldsymbol{p}) \equiv \omega_{\mathrm{L}} \int_{0}^{\infty} \mathrm{e}^{\mathrm{i} \boldsymbol{k} \cdot \Delta \boldsymbol{x}(\tau)-\mathrm{i} \omega(k) \tau} \cos \left(\omega_{\mathrm{L}} \tau\right) \mathrm{d} \tau$

where $\Delta \boldsymbol{x}(\tau)$ is the variation of the unperturbed trajectory during a time lapse $\tau$ and $\omega(k)$ the mode pulsation. This exhibits the Landau-synchrotron resonances of the form:

$R(\boldsymbol{k}, \boldsymbol{p}) \propto \delta\left[\omega(k)-k_{\|} v_{\|} \pm n \omega_{\mathrm{L}}\right]$.

For an isotropic power law spectrum $S(k) \propto \eta k^{-\beta}$,

$v_{\mathrm{s}} \sim \eta \omega_{\mathrm{L}} \rho^{\beta-1}$

where $\rho \equiv 2 \pi r_{\mathrm{L}} / L_{\max }, L_{\max }$ being the coherence length of the field. The parameter $\rho$ is the reduced rigidity and this law holds for:

$\frac{k_{\min }}{k_{\max }} \lesssim \rho \lesssim 1$.

Finally the scattering time $\tau_{\mathrm{s}} \sim v_{\mathrm{s}}^{-1}$.

In the case of strong turbulence, the gyro-resonances broaden; it turns out that the scaling law in terms of the rigidity $\rho$ and of the irregularity level $\eta$ can be extrapolated (Casse et al. 2002) when the spectrum is isotropic. In this paper, we show that the results can be different when the spectrum is anisotropic. When there is no correlation between the parallel and the perpendicular parts of the spectrum (i.e. $S_{3 \mathrm{~d}}(\boldsymbol{k}) \propto k_{\|}^{-\beta} k_{\perp}^{-q}$ with $q \equiv \alpha+1>2$ ) the result derived from the quasi-linear theory can be extrapolated to strong turbulence, giving a diffusion law similar to the isotropic case. In particular a Bohm scaling is found for $\beta=1$ (and only in that case).

The result is quite different when there is a correlation between parallel and transverse wave numbers as prescribed by 
Goldreich and Shridar, i.e. $k_{\perp} \ell_{\perp} \sim\left(k_{\|} \ell_{\|}\right)^{3 / 2}$. Now the resonance $k_{\|} r_{\mathrm{L}} \sim 1$ becomes inefficient because the requirement to have simultaneously much larger $k_{\perp}$ makes the particle insensitive to these modes (i.e. the angular scattering frequency vanishes). The numerical simulation actually indicates that the particles interact at $k_{\perp} r_{\mathrm{L}} \sim 1$, as shown in Fig. 2. This behavior is not described by the quasi-linear theory that would give a non vanishing result only if one could obtain simultaneously $k_{\|} r_{\mathrm{L}} \sim 1$.

However, if one assumes that the particle interacts with a spectrum band such that $k_{\perp} r_{\mathrm{L}} \sim 1$ and the correlation time is a few Larmor times, as is usually the case, Eq. (A.3) gives a relation of the form:

$v_{\mathrm{S}} \sim \omega_{\mathrm{L}}^{2} t_{\mathrm{L}}\left[k_{\perp}^{2} \int \frac{\mathrm{d} k_{\|}}{(2 \pi)} S_{3 \mathrm{~d}}\left(k_{\perp}, k_{\|}\right)\right]_{k_{\perp} r_{\mathrm{L}}=1}$,

which leads to a result in agreement with the numerical simulation, if one recalls the relation $3 \alpha+2 \beta=7$ (see Paper I; and Galtier et al. 2005):

$\frac{v_{\mathrm{s}}}{\omega_{\mathrm{L}}} \sim \eta \rho_{\perp}^{2 / 3}$.

This effect can be associated with a perpendicular diffusion process associated to encounters with oblique magnetic field lines encounterings. The particle angular diffusion is then controled by the perpendicular transport.

\section{Appendix B: Power loss rate per particle}

Let $\gamma_{\mathrm{r}}(\boldsymbol{k})$ be the growth rate of the resonant instability (see Paper I) and $P(\epsilon)$ the power lost by each particle of energy $\epsilon$ to trigger the turbulence spectrum $S_{3 \mathrm{~d}}(\boldsymbol{k})$. Then, these physical quantities are linked the following integrals, expressing that the power generation of waves is equal to the power loss suffered by the particles:

$\int P(\epsilon) f(p) 4 \pi p^{2} \mathrm{~d} p=-2 \int \gamma_{\mathrm{r}}(\boldsymbol{k}) S_{3 \mathrm{~d}}(\boldsymbol{k}) \frac{\mathrm{d}^{3} k}{(2 \pi)^{3}} \bar{B}^{2}$.

The growth rate is proportional to the gradient of the distribution function and depends on the pitch angle frequency $v_{\mathrm{s}}(p, \mu)$. As seen in Sect. 4, the spectrum factorizes (except in the GolreichShridar case) so that the scattering frequency is independent of the transverse spectrum and is given by

$v_{\mathrm{S}}(p, \mu)=\left.\omega_{\mathrm{L}}(p) k_{\|} S_{\|}\left(k_{\|}\right)\right|_{k_{\|}=k_{\mathrm{r}}(p, \mu)}$,

where $k_{\mathrm{r}}(p, \mu)=\left(|\mu| r_{\mathrm{L}}\right)^{-1}$. Then the growth rate is given by the following integral:

$$
\begin{aligned}
\gamma_{\mathrm{r}}\left(k_{\|}\right)= & \frac{\pi V}{8 \bar{B}^{2}} \int p^{2} \mathrm{~d} p \int \mathrm{d} \mu \frac{\omega_{\mathrm{L}}}{v_{\mathrm{s}}}\left(1-\mu^{2}\right) \\
& \times \frac{v^{2}}{c^{2}} \epsilon \delta\left(k_{\|} r_{\mathrm{L}} \mu-1\right) \frac{\partial f}{\partial x} .
\end{aligned}
$$

Inserting Eqs. (B.3) and (B.2) into Eq. (B.1), one obtains

$$
\begin{aligned}
\int P(\epsilon) f(p) 4 \pi p^{2} \mathrm{~d} p= & -\frac{V}{4} \int_{-1}^{+1}\left(1-\mu^{2}\right) \mathrm{d} \mu \\
& \times \int \frac{v^{2}}{c^{2}} \epsilon \frac{\partial f}{\partial x} 4 \pi p^{2} \mathrm{~d} p .
\end{aligned}
$$

This result is in agreement with (McKenzie \& Völk 1982) who obtained a power density for Alfvén wave generation equal to $V \partial P_{\mathrm{cr}} / \partial x$. One finally obtains the power loss per particle:

$P(\epsilon)=-\frac{1}{3} V \frac{v^{2}}{c^{2}}\left(\frac{\partial \log f}{\partial x}\right) \epsilon$

However a model of $\mathrm{CR}$ acceleration at $\mathrm{SNr}$ shock to be consistent with the CR data should include at once the amplification of turbulence upstream and the back reaction of cosmic ray on the shock structure.

\section{References}

Achterberg, A., Blandford, R. D., \& Reynolds, S. P. 1994, A\&A, 281, 220 Ballet, J. 2005, Proc. of the 35th COSPAR Symp., 765

Bamba, A., Yamazaki, R., Ueno, M., \& Koyama, K. 2003, ApJ, 589, 827

Bell, A. R. 1978, MNRAS, 182, 147

Bell, A. R. 2004, MNRAS, 353, 550

Bell, A. R., \& Lucek, S. G. 2001, MNRAS, 321, 433

Berezhko, E. G., \& Völk, H. J. 2004, A\&A, 419, L27

Berezhko, E. G., Yelshin, V. K., \& Ksenofontov, L. T. 1996, JETP, 82, 1

Berezhko, E. G., Ksenofontov, L. T., \& Völk, H. J. 2003, A\&A, 412, L11 Bykov, A. M. 2001, SSR, 99, 317

Bykov, A. M. 2004, Adv. Space Res., 33, 366

Cassam-Chenaï, G., Decourchelle, A., Ballet, J., et al. 2004, A\&A, 414, 545

Casse, F., Lemoine, M., \& Pelletier, G. 2002, Phys. Rev. D, 65, 3002

Cho, J., \& Vishniac, E. T. 2000, ApJ, 539, 273

Drury, L'O. C. 1983, Rep. Prog. Phys., 46, 973

Drury, L'O. C. 2001, American Institute of Physics (AIP) Proceedings, ed. F. A. Aharonian, \& H. J. Völk, 558, 71

Ellison, D. C., Decourchelle, A., \& Ballet, J. 2004, A\&A, 413, 189

Galtier, S., Nazarenko, S. V., Newell, A. C., \& Pouquet, A. 2000, J. Plasma Phys., 63,447

Galtier, S., Pouquet, A., \& Mangeney, A. 2005

[arXiv: physics/0504207]

Goldreich, P., \& Shridar, S. 1995, ApJ, 438, 763

Gotthelf, E. V., Halpem, J. P., Camilo, F., et al. 2001, ApJ, 552, L125

Hasselmann, K., \& Wibberenz, G. 1968, Z. Geophys., 34, 353

Hillas, A. M. 2005, J. Phys. G: Nucl. Part. Phys., 31, R95

Hughes, J. P. 1999, ApJ, 527, 298

Hughes, J. P. 2000, ApJ, 545, L53

Hwang, U., Decourchelle, A., Holt, S. S., \& Petre, R. 2002, ApJ, 581, L101

Jokipii, J. R. 1966, ApJ, 146, 180

Jokipii, J. R. 1987, ApJ, 313, 842

Jones, F. C., \& Ellison, D. C. 1991, SSR, 58, 259

Jones, T. W. 1994, ApJS, 90, 969

Kirk, J. G., Duffy, P., \& Gallant, Y. A. 1996, A\&A, 314, 1010

Lagage, P.-O., \& Césarsky, C. 1983, A\&A, 125, L249

Lemoine, M., \& Revenu, N. 2005, submitted

Long, K. S., Reynolds, S. P., Raymond, J. C., et al. 2003, ApJ, 586, 1162

Longair, M. S. 1994, High energy Astrophysics (Cambridge University press)

Lucek, S. G., \& Bell, A. R. 2000, MNRAS, 314, L65

Mc Kenzie, J. F., \& Völk, H. J. 1982, A\&A, 116, 191

Maron, J., \& Goldreich, P. 2001, ApJ, 554, 1175

Parizot, E., Marcowith, A., van der Swaluw, E., Bykov, A., \& Tatischeff, V. 2004, A\&A, 424, 747

Parizot, E., Marcowith, A., Ballet, J., \& Gallant, Y. A. 2006, A\&A, [arXiv: ph/0603723]

Pelletier, G., Lemoine, M., \& Marcowith, A. 2006, A\&A, in press [arXiv: ph/0603461] (Paper I)

Pohl, M., Yan, H., \& Lazarian, A. 2005, ApJ, 626, L101

Ptuskin, V. S., \& Zirakashvilli, V. N. 2003, A\&A, 403, 1

Ptuskin, V. S., \& Zirakashvilli, V. N. 2005, A\&A, 429, 755

Ragot, B. R. 2001, ApJ, 547, 1010

Schlickeiser, R. 2002, Cosmic-ray Astrophysics (Springer)

Vink, J. 2004, Adv. Space Sci., 33, 356

Vink, J., \& Laming, J. M. 2003, ApJ, 584, 758

Yan, H., \& Lazarian, A. 2002, Phys. Rev. Lett., 89, 281102 